\title{
Information Fusion for Local Gabor Features Based Frontal Face Verification
}

\author{
Enrique Argones Rúa ${ }^{1}$, Josef Kittler ${ }^{2}$, Jose Luis Alba Castro ${ }^{1}$, \\ and Daniel González Jiménez ${ }^{1}$ \\ 1 Signal Theory Group, Signal Theory and Communications Dep., \\ University of Vigo, 36310, Spain \\ 2 Centre for Vision, Speech and Signal Processing, \\ University of Surrey, Guildford GU2 7XH, UK
}

\begin{abstract}
We address the problem of fusion in a facial component approach to face verification. In our study the facial components are local image windows defined on a regular grid covering the face image. Gabor jets computed in each window provide face representation. A fusion architecture is proposed to combine the face verification evidence conveyed by each facial component. A novel modification of the linear discriminant analysis method is presented that improves fusion performance as well as providing a basis for feature selection. The potential of the method is demonstrated in experiments on the XM2VTS data base.
\end{abstract}

\section{Introduction}

Several studies in face recognition and verification reported in the literature suggest that the methods based on the analysis of facial components exhibit better performance than those using the full face image. There are a number of reasons that could explain this general behaviour. First of all when one deals with facial components, it should be easier to compensate for changes in illumination between gallery and probe images. Second, any pose changes can also be more readily corrected for small face patches, rather than for the whole image. Third, faces are not rigid objects and they undergo local deformations. Such deformations can seriously degrade a full image representation, but will affect only a small number of facial components. The unaffected facial components may still provide sufficient evidence about person's identity.

Although it has many advantages, the component based approach to face recognition poses a new problem. The evidence that is gathered by analysing and matching individual facial components has to be fused to a single decision. In this paper this fusion problem is addressed in the context of face verification. We propose a multistage fusion architecture and investigate several fusion methods that can be deployed at its respective stages. These include linear discriminant analysis (LDA) and multilayer perceptron (MLP). Most importantly, we propose a novel modification of the LDA fusion technique that brings two significant benefits: improved performance and considerable speed up of the face verification process. This is achieved by discarding those facial components that 
are associated with negative coefficients of the LDA projection vector. We provide some theoretical argument in support of the proposed method. Its superior performance is demonstrated by experiments on the XM2VTS database using the standard protocols.

The paper is organised as follows. In the next Section we describe the component based face representation method used in our study. Section 3 introduces the proposed fusion architecture. The novel LDA method with feature selection capabilities is presented in Section 3.2. The experiments conducted on the XM2VTS database are described and the results discussed in Section 4 . Finally, the paper is drawn to conclusion in Section 5 .

\section{Local Gabor Features for Frontal Face Verification: Local Texture Similarities}

Gabor filters are biologically motivated convolution kernels that capture the texture information and are quite invariant to the local mean brightness, so a good face encoding approach could be to extract the texture from some equally spaced windows. The local Gabor features are basically the response of several Gabor filters, with different frequencies and orientations. In this case we use 5 different frequencies and 8 different orientations, so every Gabor jet is a vector with 40 components. These Gabor jets are located in small windows which are centered following the rectangular grid pattern that we can see in the figure 1. The face images have been normalized to align the center of the eyes and the mouth to the same windows for all the images. This grid has 13 rows and 10 columns, so we have $N=130$ Gabor jets with 40 coefficients each encoding every frontal face image.

Let $\mathcal{P}=\left\{\boldsymbol{p}_{\mathbf{1}}, \boldsymbol{p}_{\mathbf{2}}, \ldots, \boldsymbol{p}_{\boldsymbol{N}}\right\}$ denote the set of points we use to extract the texture information, and $\mathcal{J}=\left\{\mathcal{J}_{\boldsymbol{p}_{1}}, \mathcal{J}_{\boldsymbol{p}_{2}}, \ldots, \mathcal{J}_{\boldsymbol{p}_{\boldsymbol{N}}}\right\}$ be the set of jets calculated for one face. The similarity function between two Gabor jets taken from two different images $\mathcal{I}^{1}$ and $\mathcal{I}^{2}$ results in:

$$
\mathcal{S}\left(\mathcal{J}_{\boldsymbol{p}_{\boldsymbol{i}}}^{1}, \mathcal{J}_{\boldsymbol{p}_{\boldsymbol{i}}}^{2}\right)=<\mathcal{J}_{\boldsymbol{p}_{\boldsymbol{i}}}^{1}, \mathcal{J}_{\boldsymbol{p}_{\boldsymbol{i}}}^{2}>
$$

where $<\mathcal{J}_{\boldsymbol{p}_{\boldsymbol{i}}}^{1}, \mathcal{J}_{\boldsymbol{p}_{\boldsymbol{i}}}^{2}>$ represents the normalized dot product between the $i$-th component from $\mathcal{J}^{1}$ and the corresponding component from $\mathcal{J}^{2}$, but taking into account that only the moduli of jet coefficients are used.

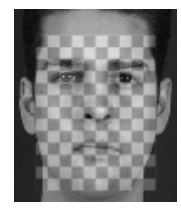

Fig. 1. Rectangular grid used to take the local features 
So, if we want to compare two frontal face images, we will get, using the equation [1, the following similarity set:

$$
\mathcal{S}_{\mathcal{I}^{1}, \mathcal{I}^{2}}=\left\{\mathcal{S}\left(\mathcal{J}_{\boldsymbol{p}_{1}}^{1}, \mathcal{J}_{\boldsymbol{p}_{1}}^{2}\right), \ldots, \mathcal{S}\left(\mathcal{J}_{\boldsymbol{p}_{\boldsymbol{N}}}^{1}, \mathcal{J}_{\boldsymbol{p}_{N}}^{2}\right)\right\}
$$

These similarity scores then have to be combined to a single decision score output by an appropriate fusion rule.

When we have $T$ training images for the client training we have several choices. One of them is to make a decision based on the similarity set that we can get comparing a single user template with the probe image. On the other hand we could use the Gabor jets of every training image as a template, and then obtain $T$ different decision scores. This approach, which is the information fusion approach adopted in this paper and is referred as multiple template method, then requires the fusion of decision scores corresponding to the individual templates.

\section{Information Fusion}

Let us suppose that we have $T$ different training images for every client. We can then build a set of $T$ decision functions for the user $k$, and we can write them as:

$$
\mathcal{D}_{i}^{k}(\mathcal{J})=f\left(\mathcal{J}, \mathcal{J}^{k, i}\right), i \in\{1, \ldots, T\},
$$

where $\mathcal{J}^{k, i}$ denotes the $i^{\text {th }}$ training image for user $k$, and assuming that the decision functions $f(\cdot)$ computed for the respective training images are identical.

As indicated in the previous Section, the decision function $\mathcal{D}_{i}^{k}(\mathcal{J})$ is realised as a two step operation where by in the first step we obtain similarity scores for the individual local jets and in the second stage we fuse these scores by a fusion rule, $g(\cdot)$, i.e.

$$
f\left(\mathcal{J}, \mathcal{J}^{k, i}\right)=g\left\{\mathcal{S}\left(\mathcal{J}_{\boldsymbol{p}_{\mathbf{1}}}, \mathcal{J}_{\boldsymbol{p}_{\mathbf{1}}}^{k, i}\right), \ldots, \mathcal{S}\left(\mathcal{J}_{\boldsymbol{p}_{\boldsymbol{N}}}, \mathcal{J}_{\boldsymbol{p}_{\boldsymbol{N}}}^{k, i}\right)\right\}
$$

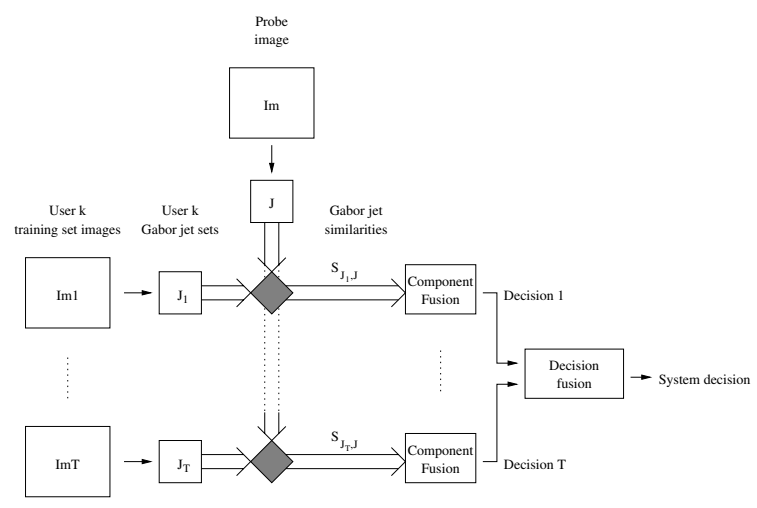

Fig. 2. Decision-fusion scheme 
The decision scores obtained for the multiple templates then have to be fused. The decision fusion function can be defined as $\mathcal{D}^{k}\left(\mathcal{D}_{1}^{k}, \ldots, \mathcal{D}_{T}^{k}\right)$, and can be performed by any suitable fusion function such as those described in the next Section 3.1. This decision fusion function must take the final decision about the identity claim as

$$
\mathcal{D}^{k}=h\left(D_{1}^{k}, \ldots, D_{T}^{k}\right)
$$

An overview of the scheme is shown in figure 2

\subsection{Fusion Methods}

The fusion of image component similarity scores defined in equation 4 as well as the decision score fusion in equation 5 can be implemented using one of several trainable or non trainable functions or rules for this task, as MLP, SVM, LDA, AdaBoost or the sum rule. For this experiment we will compare the performance of MLP and LDA. In figure 3 we can see an overview of the training and evaluation processes with these methods. Both LDA and MLP outputs are not thresholded in the decision score level because it could cause a loss of information in this stage.
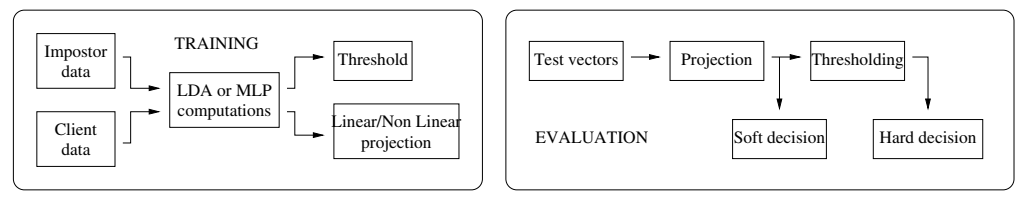

Fig. 3. LDA or MLP based fusion

The MLP that we use in this experiment is a fully connected and one hidden layer network. Based on some previous work we decided to use 3 neurons in the hidden layer to get the decision scores and 2 neurons in the hidden layer for the decision score fusion. We have trained the MLPs using the standard backpropagation algorithm.

\subsection{LDA-Based Feature Selection}

In a two class problem, LDA yields just one direction vector. Each component $v_{i}$ of the LDA vector $\mathbf{v}$ represents the weight of the contribution of the $i^{\text {th }}$ component to the separability of the two classes as measured by the eigenvalue of the LDA eigenanalysis problem. At this point it is pertinent to ask whether the coefficient values could be used to judge which of the features are least useful from the point of view of class separation. If there was a basis for identifying irrelevant features, we could reduce the dimensionality of the problem and at the same time improve the performance of the fusion system. This is the normal positive outcome one can expect from feature selection.

To answer this question, let us look at the LDA solution in more detail. Let $\mathcal{X}=$ $\left[x_{1}, \ldots, x_{N}\right]$ denote our Gabor jet similarities vector. Clearly, $x_{i}$ are not independent, as ideally, all similarity values should be high for the true identity claim and 
vice-versa for an imposter claim. However, it is not unreasonable to assume that $x_{i}$ is class conditional independent of $x_{j} \forall i, j \mid i \neq j$ and $i, j \in\{1, \ldots, N\}$. This is a relatively strong assumption, but for the sake of simplicity, we shall adopt it.

Let the mean of the $i^{\text {th }}$ component be denoted $\mu_{i, 0}=E\left\{x_{i} \mid C=0\right\}$ and $\mu_{i, 1}=E\left\{x_{i} \mid C=1\right\}$, where $C=1$ when $\mathcal{X}$ comes from a true identity claim and $C=0$ when $\mathcal{X}$ comes from a false identity claim. Let $\mu_{i}=\frac{1}{2}\left(\mu_{i, 0}+\mu_{i, 1}\right)$. Further, let $\sigma_{i, 0}^{2}=\left\{\left(x_{i}-\mu_{i, 0}\right)^{2} \mid C=0\right\}$ and $\sigma_{i, 1}^{2}=\left\{\left(x_{i}-\mu_{i, 1}\right)^{2} \mid C=1\right\}$ denote the variances of the similarity scores. Let $c_{i}=\frac{1}{2}\left(\sigma_{i, 0}^{2}+\sigma_{i, 1}^{2}\right)$.

As $x_{i}$ represents similarity and the greater the similarity the higher the value of $x_{i}$, we can assume $\mu_{i, 1}>\mu_{i, 0}, \forall i \in\{1, \ldots, N\}$.

LDA finds a one dimensional subspace in which the separability of true clients and impostors is maximised. The solution is defined in terms of the within class and between class scatter matrices $S_{w}$ and $S_{b}$ respectively, i.e.

$$
\begin{gathered}
S_{w}=\left(\begin{array}{cccc}
c_{1} & 0 & \ldots & 0 \\
0 & c_{2} & \ldots & 0 \\
\vdots & \vdots & \ddots & \vdots \\
0 & \ldots & 0 & c_{N}
\end{array}\right) \\
S_{b}=\left(\mu_{1}-\mu_{0}\right)\left(\mu_{1}-\mu_{0}\right)^{T}
\end{gathered}
$$

where $\mu_{C}$ is the mean vector of class $C$ composed of the above components.

Now the LDA subspace is defined by the solution to the eigenvalue problem

$$
S_{w}^{-1} S_{b} \mathbf{v}-\lambda \mathbf{v}=0
$$

In our face verification case equation 8 has only one non zero eigenvalue $\lambda$ and the corresponding eigenvector defines the LDA subspace. It is easy to show that the eigenvector $\mathbf{v}$ is defined as

$$
\mathbf{v}=S_{w}^{-1}\left(\mu_{1}-\mu_{0}\right)
$$

Recall that all the components of the difference of the two mean vectors are non negative. Then from equations 9 and 6 it follows that the components of the LDA vector $\mathbf{v}$ should also be non negative. If a component is non positive, it means that the actual training data is such that

- the observations do not satisfy the axiomatic properties of similarities

- the component has a strong negative correlations with some other components in the feature vector, so it is most likely encoding random redundant information emerging from the sampling problems, rather than genuine discriminatory information. Reflecting this information in the learned solution does help to get a better performance on the evaluation set where it is used as a disimilarity. However, this does not extend to the test set.

When LDA projection vector components have all the same sign, the similarity scores are re-enforcing each other and compensating for within class variations. 
But for a negative component in the projection vector a positive similarity information in that dimension is not helping to get a general solution, and it is very likely that it is being used to overfit the LDA training data.

LDA is not an obvious choice for feature selection, but in the two class case of combining similarity evidence it appears that the method offers an instrument for identifying dimensions which have an undesirable effect on fusion. By eliminating every feature with a negative projection coefficient, we obtain a lower dimensional LDA projection vector with all projection coefficients positive. This projection vector is not using many of the original similarity features, and therefore performs the role of an LDA-based feature selection algorithm.

\section{Experimental Results}

Our experiments were conducted using the XM2VTS database [1, according to the Lausanne protocol [2] in both configurations.

For verification experiments this database was divided in three different sets: training set, evaluation set (used to tune the algorithms) and test set. We have 3 different images for every client training in Configuration I of the Lausanne protocol and 4 images for every client training in Configuration II.

An important consideration about the two different configurations is that Configuration I is using the same sessions to train and tune the algorithms, so the client attempts are more correlated than in Configuration II, where the sessions used to train the algorithms are different than those used to tune the algorithms. This means that Configuration I is likely to lead to an intrinsically poorer general solution.

In tables 1 and 2 we show the single decision stage performance with and without the LDA-based feature selection. If we compare the results in both tables we can clearly draw two main conclusions:

- The TER is lower using the LDA-based feature selection for both MLP and LDA decision fusion functions in both configurations in the test set but higher in the evaluation set.

- The difference between the FAR and FRR in the test set performance is lower for both configurations and decision fusion functions.

These two suggest that the LDA-based feature selection has enabled us to construct a solution exhibiting better generalisation properties than the one obtained when using all the features together. The stability of the operating point is also better.

On the other hand, in tables 3, 4, and 5 we have the overall system performance with and without the LDA-based feature selection algorithm. If we compare the results in tables 3 and 4, where the decision fusion function is LDA (without and with the feature selection respectively) we obtain a degradation of $5.42 \%$ in TER when using the feature selection in Configuration I and an improvement of $6.71 \%$ in TER when using feature selection in Configuration II. 
Table 1. Single template performance with global thresholding and without feature selection

\begin{tabular}{|c|c|c|c|c|c|}
\cline { 3 - 6 } \multicolumn{2}{c|}{} & \multicolumn{2}{c|}{ Configuration I } & \multicolumn{2}{c|}{ Configuration II } \\
\cline { 3 - 6 } \multicolumn{2}{c|}{} & FAR(\%) & FRR(\%) & FAR(\%) & FRR(\%) \\
\hline \multirow{3}{*}{ LDA } & Ev. Set & 3.83 & 3.83 & 3.20 & 3.19 \\
\cline { 2 - 6 } & Ts. Set & 7.13 & 4.42 & 5.79 & 5.63 \\
\hline \multirow{3}{*}{ MLP } & Ev. Set & 0.90 & 0.94 & 0.76 & 0.75 \\
\cline { 2 - 6 } & Ts. Set & 2.21 & 7.42 & 2.50 & 9.50 \\
\hline
\end{tabular}

Table 2. Single template performance with LDA-based feature selection and global thresholding

\begin{tabular}{|c|c|c|c|c|c|}
\cline { 3 - 6 } \multicolumn{2}{c|}{} & \multicolumn{2}{c|}{ Configuration I } & \multicolumn{2}{c|}{ Configuration II } \\
\cline { 3 - 6 } \multicolumn{2}{c|}{} & FAR(\%) & FRR(\%) & FAR(\%) & FRR(\%) \\
\hline \multirow{3}{*}{ LDA } & Ev. Set & 4.39 & 4.39 & 3.87 & 3.87 \\
\cline { 2 - 6 } & Ts. Set & 6.79 & 4.67 & 5.44 & 5.44 \\
\hline \multirow{2}{*}{ MLP } & Ev. Set & 2.89 & 2.89 & 2.15 & 2.19 \\
\cline { 2 - 6 } & Ts. Set & 4.24 & 5.00 & 3.18 & 6.63 \\
\hline
\end{tabular}

However, if we use the MLP as the decision fusion function trained with the LDA-based feature selection features, as we can see in table 5 the results in Configuration I are much better. If we do not use feature selection prior to the MLP based similarity score fusion, the results (not listed in this paper) are much worse than those listed in table 5 for both configurations, as could be expected from the highly unbalanced results shown in table 1 for the MLP fusion method.

The overall results in Configuration I should not be considered as a reflection of the generalization power of our fusion algorithms, as the poor generalization behaviour is intrinsically imposed by the test protocol. Therefore it is reasonable to argue that the LDA-based feature selection allow us to improve the overall system performance.

Finally, the LDA-based selected features for both configurations can be seen super imposed over the face of one of the subjects of the database (for illustration purposes) in figure 4. Note that the number and location of the selected features (40 in the configuration I and 44 in the configuration II) are very simi-

Table 3. Multiple template performance using LDA without feature selection for similarity score fusion, LDA and MLP as decision fusion functions and client specific thresholding

\begin{tabular}{|c|c|c|c|c|c|}
\cline { 3 - 6 } \multicolumn{2}{c|}{} & \multicolumn{2}{c|}{ Configuration I } & \multicolumn{2}{c|}{ Configuration II } \\
\cline { 3 - 6 } \multicolumn{2}{c|}{} & FAR(\%) & FRR(\%) & FAR(\%) & FRR $(\%)$ \\
\hline \multirow{2}{*}{ LDA } & Ev. Set & 1.48 & 1.43 & 0.75 & 0.75 \\
\hline \multirow{2}{*}{ MLP } & Ts. Set & 3.39 & 3.25 & 1.92 & 2.25 \\
\cline { 2 - 6 } & Ev. Set & 1.36 & 1.33 & 0.50 & 0.50 \\
\hline
\end{tabular}


Table 4. Multiple template performance using LDA with feature selection for similarity score fusion, LDA and MLP as decision fusion functions, and client specific thresholding

\begin{tabular}{|c|c|c|c|c|c|}
\cline { 3 - 6 } \multicolumn{2}{c|}{} & \multicolumn{2}{c|}{ Configuration I } & \multicolumn{2}{c|}{ Configuration II } \\
\cline { 3 - 6 } \multicolumn{2}{c|}{} & FAR(\%) & FRR(\%) & FAR(\%) & FRR(\%) \\
\hline \multirow{2}{*}{ LDA } & Ev. Set & 1.66 & 1.67 & 0.75 & 0.75 \\
\cline { 2 - 6 } & Ts. Set & 3.75 & 3.25 & 1.89 & 2.00 \\
\hline \multirow{2}{*}{ MLP } & Ev. Set & 1.83 & 1.83 & 0.50 & 0.50 \\
\cline { 2 - 6 } & Ts. Set & 4.65 & 3.00 & 1.05 & 2.75 \\
\hline
\end{tabular}

Table 5. Multiple template performance using LDA based feature selection, MLP as similarity score fusion function, LDA and MLP as decision fusion functions and client specific thresholding

\begin{tabular}{|c|c|c|c|c|c|}
\cline { 3 - 6 } \multicolumn{2}{c|}{} & \multicolumn{2}{c|}{ Configuration I } & \multicolumn{2}{c|}{ Configuration II } \\
\cline { 3 - 6 } \multicolumn{2}{c|}{} & FAR(\%) & FRR(\%) & FAR(\%) & FRR(\%) \\
\hline \multirow{3}{*}{ LDA } & Ev. Set & 1.22 & 1.17 & 0.61 & 0.50 \\
\cline { 2 - 6 } & Ts. Set & 2.37 & 2.25 & 1.07 & 5.00 \\
\hline \multirow{2}{*}{ MLP } & Ev. Set & 1.11 & 1.00 & 0.52 & 0.50 \\
\cline { 2 - 6 } & Ts. Set & 2.20 & 2.25 & 0.93 & 8.00 \\
\hline
\end{tabular}

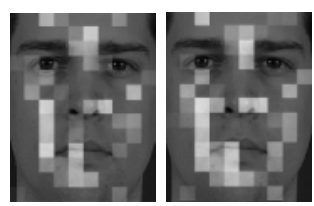

Fig. 4. LDA-based selected features for configuration I (left) and configuration II (right). The brightness is proportional to the LDA projection vector coefficient.

lar in both configurations, and even the values (represented in the figure by the window brightness) of the coefficients are also very similar. The stability and consistency of the features identified by the proposed algorithm is very encouraging. Moreover, the number of selected features is small enough to allow a high reduction in the computational complexity in the verification phase, and hence an important reduction (nearly a $60 \%$ ) in the verification time.

\section{Conclusions}

We addressed the problem of information fusion in component based face verification where similarity scores computed for individual facial components have to be combined to reach a final decision. We proposed a multistage fusion architecture and investigated several fusion methods that could be deployed at its respective stages. These included LDA and MLP. Most importantly, we proposed a novel modification of the LDA fusion technique that brings two significant 
benefits: improved performance and considerable speed up of the face verification process. This was achieved by discarding those facial components that were associated with negative coefficients of the LDA projection vector. We provided some theoretical argument in support of the proposed method. Its superior performance was demonstrated by experiments on the XM2VTS database using the standard protocols. Performance improvements, on the more realistic Configuration II, varying between $7-20 \%$ were achieved with the proposed method.

\section{References}

1. K. Messer, J. Matas, J. Kittler, J. Luettin and G. Maître: XM2VTSDB: The extended M2VTSDB. International Conference on Audio and Video-based Biometric Person Authentication, 1999

2. J. Luettin and G. Maître: Evaluation protocol for the XM2FDB (Lausanne protocol). IDIAP Communication, 1998.

3. Wiskott, L., Fellous, J.M., Kruger, N. and von der Malsburg, C.: Face recognition by Elastic Bunch Graph Matching. IEEE Transactions on Pattern Analysis and Machine Intelligence, 19(7), 775-779, 1997

4. L. I. Kuncheva: "Fuzzy" versus "Nonfuzzy" in combining classifiers designed by boosting. IEEE Transactions on Fuzzy Systems, 11(6), 729-741, 2003

5. P. Silapachote, Deepak R. Karuppiah, and Allen R. Hanson: Feature selection using AdaBoost for face expression recognition. Proceedings of the Fourth IASTED International Conference on Visualization, Imaging, and Image Processing, 84-89, 2004

6. P. Viola and M. Jones: Robust Real-Time Face Detection. International Conference on Computer Vision, 2001

7. B. Heisele, P. Ho and T. Poggio: Face Recognition with Support Vector Machines: Global versus Component-based Approach. International Conference on Computer Vision, 2001

8. A. Tefas, C. Kotropoulos and Ioannis Pitas: Face verification using elastic graph matching based on morphological signal decomposition. Signal Processing 82(6), 833-851, 2002

9. R. Brunelli and T. Poggio. Face Recognition: Features versus Templates. IEEE Transactions on Pattern Analysis and Machine Intelligence, 15(10), 1042-1052, O1993

10. K. Jonsson, J. Kittler, Y. P. Li and J. Matas: Learning Support Vectors for Face Verification and Recognition. Proceedings of the Fourth IEEE International Conference on Automatic Face and Gesture Recognition, 2000

11. C. Sanderson and K.K. Paliwal: Fast feature extraction method for robust face verification. Electronics Letters Online No: 20021186, 2002

12. M. Saban and C. Sanderson: On Local Features for Face Verification. IDIAP-RR, 36,2004

13. C. Havran, L. Hupet, J. Czyz, J. Lee, L. Vandendorpe, M. Verleysen: Independent Component Analysis for face authentication. Knowledge-Based Intelligent Information and Engineering Systems, 1207-1211, 2002

14. K. Messer, J. Kittler, M. Sadeghi, M. Hamouz, A. Kostyn, S. Marcel, S. Bengio, F. Cardinaux, C. Sanderson, N. Poh, Y. Rodriguez, K. Kryszczuk, J. Czyz, L. Vandendorpe, J. Ng, H. Cheung, and B. Tang: Face Authentication Competition on the BANCA Database. 